\title{
The Numerical Study of an Electron Stream Relaxation in a Non-Uniform Plasma
}

\author{
E. A. Zverev and V. G. Ledenev \\ Institute of Solar-Terrestrial Physics SD RAS, P.O.Box 4026, 664033, Irkutsk, Russia, email: \\ leden@iszf.irk.ru
}

The one-dimensional relaxation of a spatially bounded electron stream in non-uniform background plasma is simulated. The corresponding system of quasi-linear equations is

$$
\frac{\partial f}{\partial t}+V \frac{\partial f}{\partial x}=\frac{\partial}{\partial V}\left(D \frac{\partial f}{\partial V}\right), \frac{\partial W}{\partial t}=2 \gamma W, \gamma=\frac{\pi}{2} \frac{\omega_{p}}{n_{p}} V^{2} \frac{\partial f}{\partial V}, D=\frac{8 \pi^{2} e^{2}}{m^{2}} \frac{W}{V} .
$$

Here $f$ - the electron distribution function, $D$ - diffusion coefficient, and $\gamma$ and $W$ - are the growth rate and spectral energy density of plasma waves respectively, $n$ - background plasma density, $\omega_{p}$ - plasma frequency. Initial conditions (the electron distribution function at $t=0)$ :

$$
f(x, V, t=0)=A n_{b} \exp \left(-\frac{\left(V-V_{b}\right)^{2}}{2 V_{T b}^{2}}\right) \exp \left(-\left(\frac{x}{d}\right)^{2}\right)
$$

Here $n_{b}$ - electron density at $x=0, d$ - stream length, $A$ - constant. In ours simulations we have chosen: $d=10^{7} V_{T} / \omega_{p 0}, V_{b}=20 V_{T}, V_{T b}=V_{T}$, where $V_{T}$ - plasma thermal velocity. The used in calculations initial density range $-n_{b} / n_{0}=10^{-7} \div 10^{-4}$, where $n_{0}$ - the background plasma density at $x=0$. The simulation for case of non-uniform medium was performed. Background plasma density profile along the stream propagation was chosen as: $n(x)=n_{0} \exp (-x / L)$, where $L=50 d$.

The results of quasi-linear system solution are shown in Figures 1, 2. The initial electron distribution is in Figure 1. At the right the plasma waves spectral energy density (upper panels) and electron distribution (lower contours) at different time $\tau$ for the stream of initial density $n_{b} / n_{0}=10^{-4}$ propagating in non-uniform background plasma are shown. The time is given on the lower pictures, and on upper pictures the value of the moving (right) maximum of the spectral density is shown. In Figure 2 the same is shown for initial stream density $n_{b} / n_{0}=10^{-7}$. One can see that there is full relaxation in velocity space and weak widening in coordinate space for dense electron stream (Figure 1). For weaker electron stream we have mainly widening in coordinate space (Figure 2).

We suppose that the emission spectrum is determined by plasma wave energy density to the two power. The emission dynamic spectra are shown for $n_{b} / n_{0}=10^{-7}, 10^{-6}, 10^{-5}, 10^{-4}$ in Figures 3(a-d), respectively. One can see from the Figures that the emission source velocity is constant and the spectrum is the narrower the higher stream density.

\section{Acknowledgements}

This work was supported by RFBR grants 03-02-16229, 477.2003.2. 

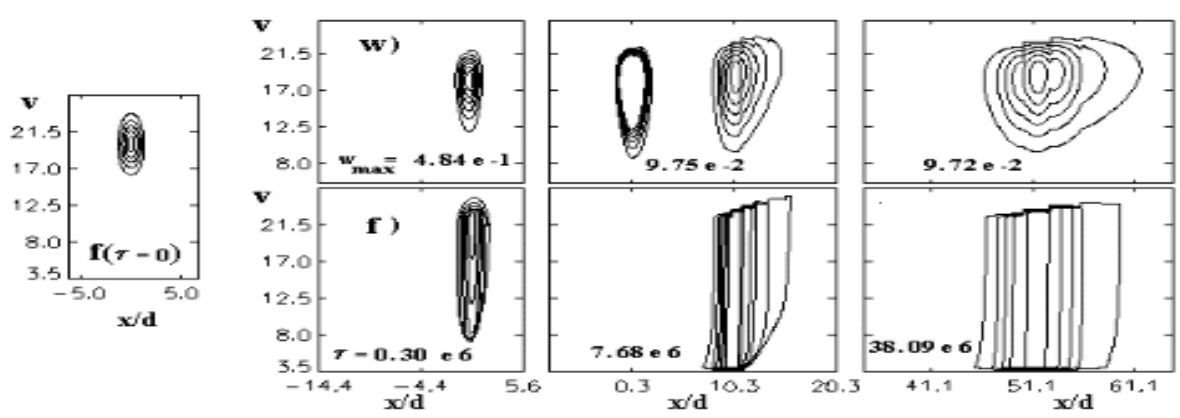

Figure 1.
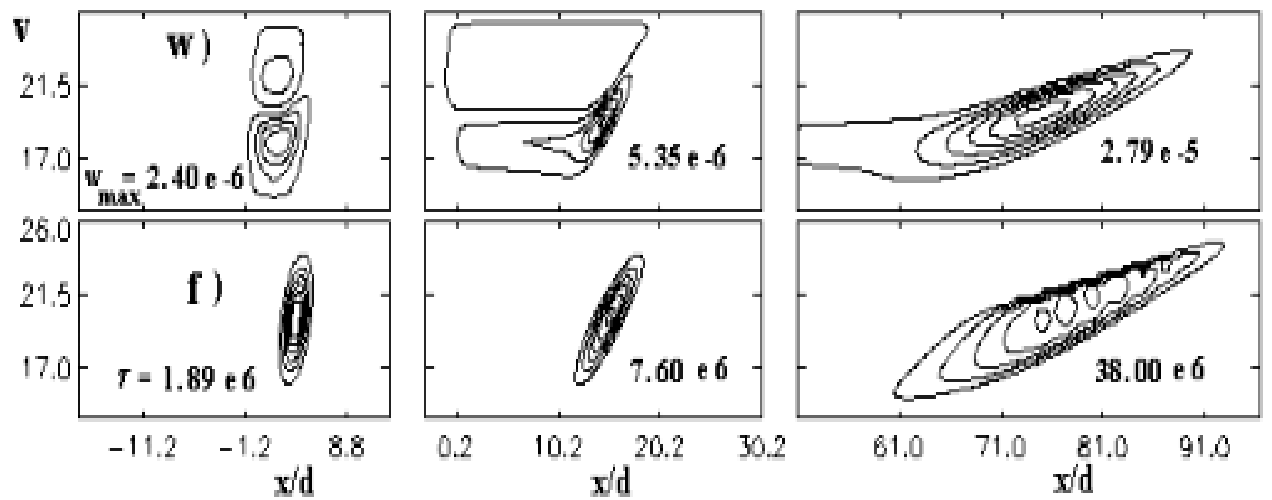

Figure 2.
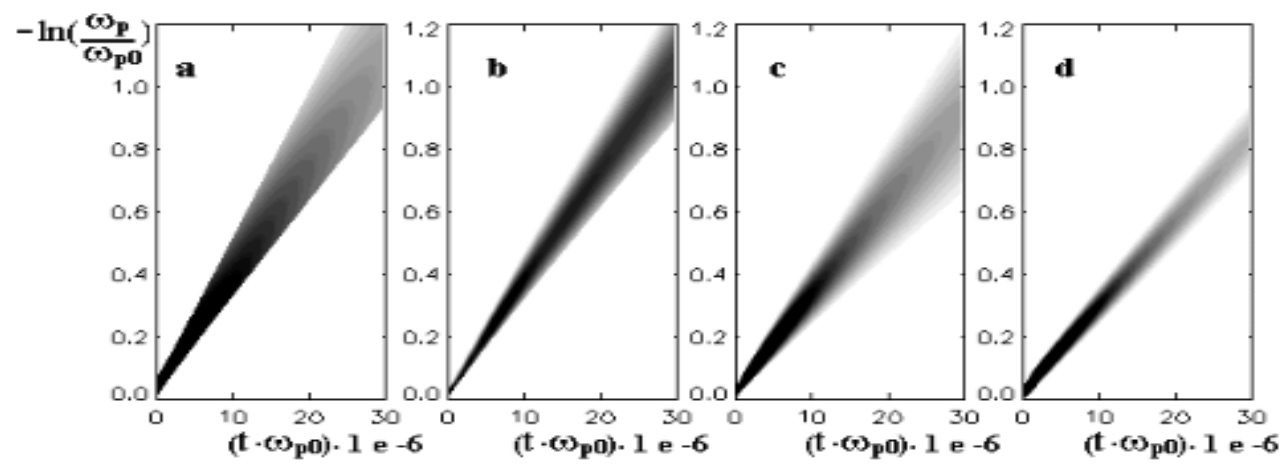

Figure 3. 